\title{
Prevalence of Atypical Pathogens in Patients With Cough and Community-Acquired Pneumonia: A Meta-Analysis
}

\author{
Cbristian Marchello, MS, MT \\ (ASCP) \\ Ariella Perry Dale, MPH \\ Thuy Nbu Thai, BS \\ Duk Soo Han, RN \\ Mark H. Ebell, MD, MS \\ Department of Epidemiology and \\ Biostatistics, College of Public Health, \\ University of Georgia, Athens, Georgia
}

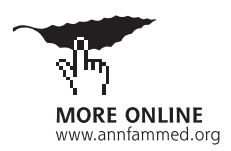

Conflicts of interest: authors report none.

\section{CORRESPONDING AUTHOR}

Mark H. Ebell MD, MS

125 Miller Hall

UGA Health Sciences Campus

Athens, GA 30602

ebell@uga.edu

\begin{abstract}
PURPOSE Community-acquired pneumonia (CAP), acute cough, bronchitis, and lower respiratory tract infections (LRTI) are often caused by infections with viruses or Streptococcus pneumoniae. The prevalence of atypical pathogens Mycoplasma pneumoniae, Chlamydophila pneumoniae, Legionella pneumophila, and Bordetella pertussis among patients with these illnesses in the ambulatory setting has not been previously summarized. We set out to derive prevalence information from the existing literature.
\end{abstract}

METHODS We performed a systematic review of MEDLINE for prospective, consecutive-series studies reporting the prevalence of $M$ pneumoniae, C pneumoniae, $L$ pneumophila and/or B pertussis in outpatients with cough, acute bronchitis, LRTI, or CAP. Articles were independently reviewed by 2 authors for inclusion and abstraction of data; discrepancies were resolved by consensus discussion. A meta-analysis was performed on each pathogen to calculate the pooled prevalence estimates using a random effects model of raw proportions.

RESULTS Fifty studies met our inclusion criteria. While calculated heterogeneity was high, most studies reported prevalence for each pathogen within a fairly narrow range. In patients with CAP, the overall prevalences of $M$ pneumoniae and C pneumoniae were 10.1\% (95\% Cl, 7.1\%-13.1\%) and 3.5\% (95\% Cl, 2.2\%-4.9\%), respectively. Consistent with previous reports, $M$ pneumoniae prevalence peaked in roughly 6-year intervals. Overall prevalence of $L$ pneumophila was $2.7 \%(95 \%$ $\mathrm{Cl}, 2.0 \%-3.4 \%$ ), but the organism was rare in children, with only 1 case in 1,765. In patients with prolonged cough in primary care, the prevalence of $B$ pertussis was $12.4 \%(95 \% \mathrm{Cl}, 4.9 \%-19.8 \%)$, although it was higher in studies that included only children (17.6\%; $95 \% \mathrm{Cl}, 3.4 \%-31.8 \%)$.

CONCLUSIONS Atypical bacterial pathogens are relatively common causes of lower respiratory diseases, including cough, bronchitis, and CAP. Where surveillance data were available, we found higher prevalences in studies where all patients are tested for these pathogens. It is likely that these conditions are underreported, underdiagnosed, and undertreated in current clinical practice.

Ann Fam Med 2016;14:552-566. doi: 10.1370/afm.1993.

\section{INTRODUCTION}


ough is the 4 th most common reason for an office visit to an ambulatory physician, accounting for $2.8 \%$ of all visits. ${ }^{1}$ In primary care, when cough is the patient's primary complaint, it is most often caused by a virus, but approximately $5 \%$ of patients have community-acquired pneumonia (CAP). ${ }^{2}$ Although viruses and Streptococcus pneumoniae are the most common causes of $\mathrm{CAP}$, some episodes are caused by an atypical bacterial infection such as Mycoplasma pneumoniae, Cblamydopbila pneumoniae (also known as Chlamydia pneumoniae), and Legionella pneumopbila. Some episodes of non-pneumonia lower respiratory tract infection (LRTI) are caused by the above pathogens as well as by Bordetella pertussis, and the incidence of the latter is increasing in the United States. ${ }^{3}$ 
Mycoplasma pneumoniae infection is thought to vary cyclically, 4,5 and has been the cause of outbreaks of LRTI. ${ }^{6}$ Not to be confused with Chlamydia psittaci (which also causes respiratory infections but is contracted from birds), Cblamydopbila pneumoniae is more common in children, but has been associated with subsequent serious adult disease as well. A meta-analysis reported an association with lung cancer in patients with previous $C$ pneumoniae infections, ${ }^{7}$ while others have posited an association with development of asthma. ${ }^{8,9}$ Legionellosis, better known as Legionnaires' disease, is caused by L pneumopbila and is most commonly diagnosed as a cause of CAP in patients over 50 years of age, and more often in men than women. The organism is found naturally in the environment, and the infection is associated with inhalation of aerosolized water from sources such as hot tubs and cooling towers. ${ }^{10}$ Recently, increased risk of infection with $L$ pneumophila has also been linked to wet, humid weather. ${ }^{11}$ Bordetella pertussis is highly communicable and is a source of significant morbidity in children and prolonged symptoms in all patients. Although $B$ pertussis is the only atypical pathogen to have a widely available vaccine, the incidence of $B$ pertussis in the United States is increasing, with more cases in 2012 than any year previously since $1955 .^{3}$

The prevalence of atypical pathogens, particularly in the outpatient primary care setting, has not been previously summarized. B pertussis and L pneumopbila are reported by national surveillance systems in many countries, but they are laboratory-based systems that are subject to significant underreporting. ${ }^{12}$ The prevalence of $C$ pneumoniae and $M$ pneumoniae vary widely in previous studies of patients with CAP.

Because these atypical pathogens do not respond to beta-lactams, may carry a different prognosis, and can cause serious complications in some patients, it is important to understand their prevalence. Therefore, we performed a meta-analysis to describe the prevalence of atypical pathogens among 2 groups: patients with cough, acute bronchitis, or LRTI in the ambulatory setting and patients diagnosed with CAP. We also compared these "real world" prevalences with the prevalences reported by surveillance systems, where available.

\section{METHODS}

\section{Literature Review}

We searched MEDLINE for prospective studies that reported the results of testing for $M$ pneumoniae, $C$ pneumoniae, $L$ pneumophila, or B pertussis in outpatients with cough, acute bronchitis, or LRTI, as well as among inpatients and outpatients diagnosed with CAP. In order to reflect contemporary prevalences and microbiology, searches were limited to articles where the majority of data was collected after January 1, 2000. We included articles with abstracts written in English and German (the primary languages of the investigators). Supplemental Appendix A (http://www. annfammed.org/content/14/6/552/suppl/DC1) includes detailed search terms used for each strategy. We also reviewed the reference lists for review articles identified by our search, and of any included studies.

We excluded studies of only or predominantly immunocompromised patients, studies of hospital-acquired infections, studies of special or unusual populations (eg, military recruits), studies of acute exacerbations of chronic obstructive pulmonary disease or asthma, and studies of the etiology of bronchiolitis. Further, we excluded studies set in low- or medium-income countries based on Organisation for Economic Cooperation and Development (OECD) criteria; (Supplemental Appendix B, http://www.annfammed.org/content/14/6/552/suppl/ DC1) since we felt that they would not reflect the current practice and epidemiology of the United States. We also excluded case-control studies, case reports, case series and retrospective studies, outbreak investigations, and studies that did not use culture, polymerase chain reaction $(\mathrm{PCR}$ ), serology, or urine antigen testing (for $L$ pneumopbila) to identify pathogens.

\section{Data Abstraction}

Two investigators reviewed each abstract to identify articles that should be reviewed in full. Any article selected for full review was examined by both investigators. For each included article, study characteristics and data regarding prevalence were abstracted by both authors. For prevalence data, definite and probable cases were included and possible cases were excluded. Any discrepancies were resolved by consensus discussion.

\section{Surveillance Systems}

We used surveillance data reported by high-income members of the OECD. ${ }^{13}$ The most recent complete data available, from 2012, were abstracted by 2 investigators, with any discrepancies resolved by consensus discussion. For each report, we documented the type of surveillance used, number of cases reported, and total population.

\section{Study Quality}

A meta-analysis usually uses a standardized tool to assess the risk of bias. ${ }^{14-16}$ Unfortunately, there are currently no published tools for assessing bias in studies of disease prevalence. To ensure that the studies included in our meta-analysis were of consistent high quality, we only included studies that met the following criteria: they enrolled consecutive patients, did not gather data from a specialized or unusual population, gathered data 
prospectively, and used diagnostic tests likely to classify patients accurately as having the pathogen in question.

\section{Analysis}

We identified 2 groups for the analysis: patients presenting with acute cough illness or lower respiratory tract symptoms and patients diagnosed with CAP. Where studies reported etiology separately for patients with CAP and those with non-pneumonia LRTI, we report these groups separately as well.
Pooled prevalence estimates were calculated with random effects model of raw proportions. Statistical analysis was performed in $\mathrm{R}$ (version 3.2.2, R Studio Version 0.99 .441 ), including plots of proportions with each pathogen using the metafor procedure.

\section{RESULTS}

The search for $M$ pneumoniae, C pneumoniae, and L pneumopbila yielded 449 abstracts. A separate search for

\section{Figure 1. PRISMA diagram.}

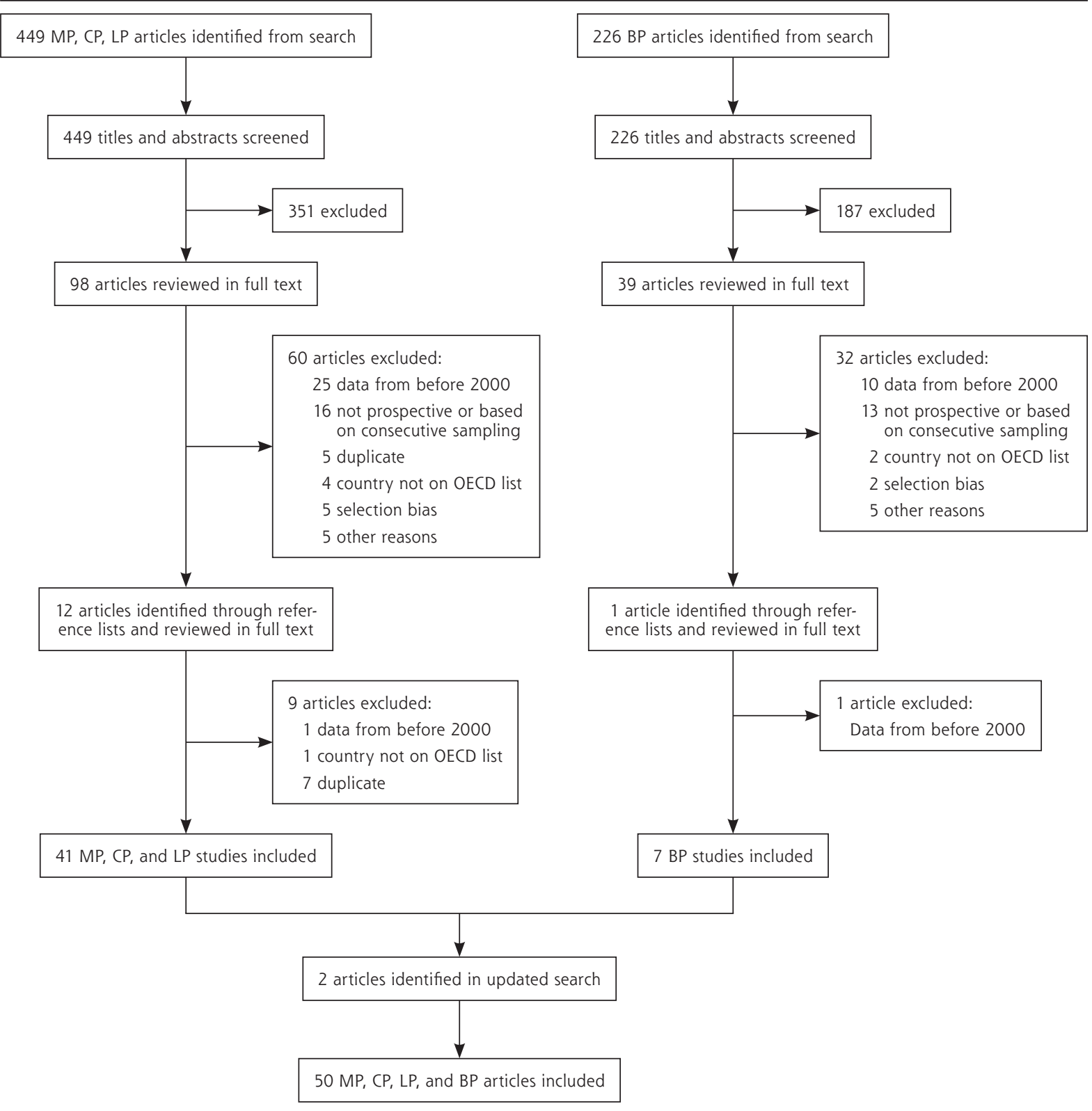

$\mathrm{BP}=$ Bordetella pertussis; $\mathrm{CP}=$ Chlamydophila pneumoniae; $\mathrm{LP}=$ Legionella pneumophila; $\mathrm{MP}=$ Mycoplasma pneumonia; $\mathrm{OECD}=$ Organization for Economic Cooperation and Development. 
$B$ pertussis returned 226. After screening titles and abstracts, 98 articles for $M$ pneumoniae, C pneumoniae, and $L$ pneumopbila and 39 for $B$ pertussis remained for full-text review. Thirteen articles were additionally identified through a review of the reference lists (12 for $M$ pneumoniae, C pneumoniae, and L pneumopbila, and 1 for $B$ pertussis). Full-text review excluded 102 articles. The most common reasons for exclusion were that the majority of data was collected before 2000 or that the study did not use a cohort design with prospective data collection. An updated search before writing yielded 2 additional studies ${ }^{17,18}$ for a final of 50 included studies (Figure 1).

To compare the prevalences given in the identified
Table 1. Reported Bordetella pertussis and Legionella pneumophila Prevalence in 2012 by Case-Based Surveillance Systems of HighIncome Countries Belonging to the OECD

\begin{tabular}{|c|c|c|c|c|c|}
\hline Country ${ }^{a}$ & $\begin{array}{c}\text { BP } \\
\text { Cases }\end{array}$ & $\begin{array}{c}\text { LP } \\
\text { Cases }\end{array}$ & Population ${ }^{b}$ & $\begin{array}{c}\text { BP Rate } \\
\text { per } 100,000\end{array}$ & $\begin{array}{c}\text { LP Rate } \\
\text { per } 100,000\end{array}$ \\
\hline Australia & 24,069 & 382 & $22,918,688$ & 105.0 & 1.67 \\
\hline Austria & 425 & 101 & $8,428,915$ & 5.0 & 1.20 \\
\hline Belgium & ND & 106 & $10,787,788$ & ND & 0.98 \\
\hline Canada & 4,540 & 483 & $34,674,708$ & 13.1 & 1.39 \\
\hline Chile & 4,237 & ND & $17,423,214$ & 24.3 & ND \\
\hline Czech Republic & 707 & 56 & $10,565,678$ & 6.7 & 0.53 \\
\hline Denmark & 1,136 & 127 & $5,592,738$ & 20.3 & 2.27 \\
\hline Estonia & 149 & 3 & $1,339,762$ & 11.1 & 0.22 \\
\hline Finland & 541 & 10 & $5,402,627$ & 10.0 & 0.19 \\
\hline France & ND & 1,298 & $63,457,777$ & ND & 2.05 \\
\hline Germany & ND & 628 & $81,990,837$ & ND & 0.33 \\
\hline Greece & 40 & 27 & $11,418,878$ & 0.35 & 0.77 \\
\hline Hungary & 5 & 33 & $9,949,589$ & 0.05 & 0.24 \\
\hline Iceland & 36 & 2 & 328,290 & 11.0 & 0.61 \\
\hline Ireland & 264 & 15 & $4,579,498$ & 5.8 & 0.33 \\
\hline Italy & 262 & 1,332 & $60,964,145$ & 0.43 & 2.18 \\
\hline Japan & ND & 903 & $126,434,653$ & ND & 0.71 \\
\hline Korea, Rep. & 126 & 25 & $48,588,326$ & 0.26 & 0.05 \\
\hline Luxembourg & 11 & 5 & 523,362 & 2.1 & 0.96 \\
\hline Netherlands & 12,868 & 304 & $16,714,228$ & 77.0 & 1.82 \\
\hline New Zealand & 2,320 & 152 & $4,461,257$ & 52.0 & 3.41 \\
\hline Norway & 4,243 & 25 & $4,960,482$ & 85.5 & 0.50 \\
\hline Polandc & 1,824 & 8 & $38,317,090$ & 4.8 & 0.02 \\
\hline Portugal & 230 & 140 & $10,699,333$ & 2.1 & 1.31 \\
\hline Slovak Republic & 917 & 4 & $5,480,332$ & 16.7 & 0.07 \\
\hline Slovenia & 153 & 82 & $2,040,057$ & 7.5 & 4.02 \\
\hline Spain & 1,565 & 972 & $46,771,596$ & 3.3 & 2.08 \\
\hline Sweden & 279 & 12 & $9,495,392$ & 2.9 & 0.13 \\
\hline Switzerland & ND & 91 & 7,733,709 & ND & 1.18 \\
\hline United Kingdom & 11,993 & 401 & $62,798,099$ & 19.1 & 0.64 \\
\hline United States & 48,277 & 3,688 & $315,791,284$ & 15.3 & 1.17 \\
\hline $\begin{array}{l}\mathrm{BP}=\text { Bordetella pertu } \\
\text { eration and Develop } \\
\text { a No data available } \\
\text { b Population based } \\
\text { ' Poland used aggre }\end{array}$ & $\begin{array}{l}\text { is; } L P=L e c \\
\text { lent. } \\
\text { Israel. } \\
\text { Gapminde } \\
\text { ated instea }\end{array}$ & $2012 .^{19}$ & ophila; ND = no dat & ; OECD = Organisati & for Economic Coop \\
\hline
\end{tabular}

studies with the prevalences from surveillance systems, we abstracted surveillance data for reported cases of B pertussis and L pneumophila in 2012. Data, which were available for 31 of the 32 high-income member countries of the OECD, are summarized in Table 1 (Israel did not provide any publicly accessible data.)

\section{Prevalence of Mycoplasma pneumoniae, Chlamydophila pneumonia, and Legionella pneumophila}

A total of 30 studies reported the prevalence of $M$ pneumoniae, C pneumoniae, or L pneumophila in adults, ${ }_{1}^{18,20-48}$ and 10 studies reported the prevalence of these pathogens in children ${ }^{49-58}$ (Table 2). Only 2 studies were set in the United States. ${ }^{18,53}$

\section{Patients With Community- Acquired Pneumonia}

Figures 2-4 show the forest plots for $M$ pneumoniae, C pneumoniae, and $L$ pneumopbila respectively in patients with CAP. The overall prevalence of $M$ pneumoniae was $10.1 \%$ (95\% CI, 7.1\%-13.1\%). The prevalence was higher in children $(17.6 \%$; $95 \% \mathrm{CI}, 8.7 \%-26.4 \%)$ than in adults $(7.2 \% ; 95 \% \mathrm{CI}$, $5.2 \%-9.3 \%)$. There was significant heterogeneity, though, especially in studies of children. This is likely because outbreaks of $M$ pneumoniae are thought to occur every 4 to 6 years, and inspection of the forest plot, which is sorted chronologically, does reveal peaks around 2004 and 2010. ${ }^{62,63}$

The overall prevalence of C pneumoniae in patients with CAP was $3.5 \%$ (95\% CI, 2.2\%-4.9\%). Infection with $\mathrm{C}$ pneumoniae was more common in adults $(4.3 \%$, $95 \% \mathrm{CI}, 2.4 \%-6.2 \%)$ than in children $(1.0 \%, 95 \% \mathrm{CI}, 0.6 \%-1.5 \%)$. There was significant heterogeneity, although only 4 of 25 studies in adults had a prevalence greater than $10 \%$, while the remainder had a prevalence between $0.3 \%$ and $7.7 \%$. In children, only 2 of 10 studies had prevalences greater than $5 \%$, while the remaining 8 had prevalences ranging from $0.5 \%$ to $2.7 \%$. We reviewed the 6 identified outliers, but were 
Table 2. Characteristics of Studies of the Prevalence of Mycoplasma pneumoniae, Chlamydia pneumoniae, and Legionella pneumophila in Patients With Community-Acquired Pneumonia or Lower Respiratory Tract Infection

\begin{tabular}{|c|c|c|c|c|c|c|c|}
\hline $\begin{array}{l}\text { Author, Year } \\
\text { (Country) }\end{array}$ & Population & $\begin{array}{l}\text { Totall } \\
\text { Confirmed } \\
\text { Cases }^{a}\end{array}$ & Setting & Age & Pathogen & $\begin{array}{l}\text { Data } \\
\text { Collection } \\
\text { Period }\end{array}$ & $\begin{array}{c}\text { Diagnostic } \\
\text { Method }\end{array}$ \\
\hline \multicolumn{8}{|l|}{ CAP in Adults } \\
\hline $\begin{array}{l}\text { Jain et al, }{ }^{18} 2015^{b} \\
\text { (United States) }\end{array}$ & $\begin{array}{l}\text { Adults } \geq 18 \text { y } \\
\text { with CAP }\end{array}$ & $2,320 / 853$ & Inpatient & Median $57 \mathrm{y}$ & $M P, C P, L P$ & $2010-2012$ & $\begin{array}{l}\text { PCR, Culture, } \\
\text { UA }\end{array}$ \\
\hline $\begin{array}{l}\text { Angeles et al, }{ }^{20} 2006 \\
\text { (Spain) }\end{array}$ & $\begin{array}{l}\text { Adults } \geq 15 \text { y } \\
\text { with CAP }\end{array}$ & 198/112 & Inpatient & Median 70 y & $M P, C P, L P$ & $2003-2004$ & Serology, UA \\
\hline $\begin{array}{l}\text { Beović et al, }{ }^{21} 2003 \\
\text { (Slovenia) }\end{array}$ & $\begin{array}{l}\text { Adults } \geq 15 \text { y with } \\
\text { CAP (PSI }=1 \text { or II) }\end{array}$ & $113 / 68$ & $N R$ & Mean 44.9 y & $M P, C P, L P$ & 1999-2001 & Serology \\
\hline $\begin{array}{l}\text { Charles et al,22 } 2008 \\
\text { (Australia) }\end{array}$ & $\begin{array}{l}\text { Adults } \geq 18 \text { y } \\
\text { with CAP }\end{array}$ & $885 / 404$ & Inpatient & $\begin{array}{c}\text { Mean } 65.1 y_{1} \\
\text { range } 18 y-100 y)\end{array}$ & $M P, C P, L P$ & $2004-2006$ & Serology, UA \\
\hline $\begin{array}{l}\text { Cilloniz et al, }{ }^{23} 2012 \\
\text { (Spain) }\end{array}$ & $\begin{array}{l}\text { Adults } \geq 16 \text { y } \\
\text { with CAP }\end{array}$ & $568 / 188$ & Outpatient & Mean 47.2 y & $M P, C P, L P$ & $2000-2010$ & Serology, UA \\
\hline $\begin{array}{l}\text { Diaz et al, }{ }^{24} 2007 \\
\text { (Chile) }\end{array}$ & $\begin{array}{c}\text { Adults } \geq 16 \text { y } \\
\text { with CAP }\end{array}$ & $176 / 98$ & Inpatient & $\begin{array}{l}\text { Mean } 65.8 y_{1} \\
\text { range } 17 y-101 y\end{array}$ & $M P, C P, L P$ & $2003-2005$ & Serology, UA \\
\hline $\begin{array}{l}\text { Espana et al, }{ }^{25} 2012 \\
\text { (Spain) }\end{array}$ & $\begin{array}{l}\text { Adults } \geq 18 \text { y } \\
\text { with CAP }\end{array}$ & $344 / 153$ & $\begin{array}{l}73 \text { Inpatient, } \\
271 \text { outpatient }\end{array}$ & Mean 53.5 y & $M P, C P, L P$ & $2006-2007$ & Serology, UA \\
\hline $\begin{array}{l}\text { Falguera et al, }{ }^{26} 2010 \\
\text { (Spain) }\end{array}$ & $\begin{array}{l}\text { Adults } \geq 18 \text { y or } \\
\text { older with CAP } \\
\text { (PSI IV or V) }\end{array}$ & $88 / 25$ & Inpatient & Mean 64 y & LP & $2006-2008$ & Serology, UA \\
\hline $\begin{array}{l}\text { Gutierrez et al, }{ }^{27} 2006 \\
\text { (Spain) }\end{array}$ & $\begin{array}{l}\text { Adults } \geq 15 \text { y } \\
\text { with CAP }\end{array}$ & $493 / 250$ & $\begin{array}{l}361 \text { Inpatient, } \\
132 \text { outpatient }\end{array}$ & $\begin{array}{l}\text { Mean } 56.6 y \\
\text { range } 15 y-94 \text { y }\end{array}$ & $M P, C P, L P$ & 1999-2001 & Serology, UA \\
\hline $\begin{array}{l}\text { Herrera-Lara et al, }{ }^{28} \\
2013 \text { (Spain) }\end{array}$ & $\begin{array}{l}\text { Adults } \geq 18 \text { y } \\
\text { with CAP }\end{array}$ & $243 / 139$ & Inpatient & Mean 63.9 y & $M P, C P, L P$ & 2006-2009 & Serology, UA \\
\hline $\begin{array}{l}\text { Holm et } \mathrm{al}_{,}^{29} 2007^{\mathrm{b}} \\
\text { (Denmark) }\end{array}$ & $\begin{array}{l}\text { Adults } \geq 18 \text { y } \\
\text { with CAP }\end{array}$ & $48 / 21$ & $\begin{array}{l}9 \text { Inpatient, } \\
39 \text { outpatient }\end{array}$ & $\begin{array}{l}\text { Mean } 61 \text { y, } \\
\text { range } 22 y-88 \text { y }\end{array}$ & $M P, C P, L P$ & $2002-2003$ & PCR \\
\hline $\begin{array}{l}\text { Huijskens et al, }{ }^{30} 2013 \\
\text { (Netherlands) }\end{array}$ & $\begin{array}{l}\text { Adults } \geq 20 \text { y } \\
\text { with CAP }\end{array}$ & $408 / 263$ & NR & $\begin{array}{l}\text { Mean } 65 y_{1} \\
\text { range } 20 y-94 \text { y }\end{array}$ & $M P, C P, L P$ & $2008-2009$ & $\begin{array}{l}\text { Serology, } \\
P C R, U A\end{array}$ \\
\hline $\begin{array}{l}\text { Johansson et al, }{ }^{31} 2010 \\
\text { (Sweden) }\end{array}$ & $\begin{array}{c}\text { Adults } \geq 18 \text { y } \\
\text { with CAP }\end{array}$ & $184 / 124$ & Inpatient & $\begin{array}{l}\text { Mean } 61.3 y_{1} \\
\text { range } 18 y-93 y\end{array}$ & $M P, C P, L P$ & $2004-2005$ & $\begin{array}{l}\text { Serology, } \\
P C R, U A\end{array}$ \\
\hline $\begin{array}{l}\text { Lee et al, }{ }^{32} 2002 \\
\text { (South Korea) }\end{array}$ & $\begin{array}{c}\text { Adults } \geq 16 \text { y } \\
\text { with CAP }\end{array}$ & $81 / 15$ & Inpatient & $\begin{array}{l}\text { Mean } 66.3 y_{1} \\
\text { range } 17 y-92 y\end{array}$ & $M P, C P, L P$ & 1999-2000 & Serology \\
\hline $\begin{array}{l}\text { Luchsinger et al, }{ }^{33} \\
2013 \text { (Chile) }\end{array}$ & $\begin{array}{c}\text { Adults } \geq 18 \text { y } \\
\text { with CAP }\end{array}$ & $356 / 232$ & $\begin{array}{l}330 \text { Inpatient, } \\
26 \text { outpatient }\end{array}$ & Mean $59.3 y^{c}$ & $M P, C P, L P$ & $2005-2007$ & $\begin{array}{l}\text { Serology, } \\
\text { PCR, UA }\end{array}$ \\
\hline $\begin{array}{l}\text { Marrie et al, }{ }^{34} 2005 \\
\text { (Canada) }\end{array}$ & $\begin{array}{l}\text { Adults } \geq 18 \text { y } \\
\text { with CAP }\end{array}$ & $507 / 245$ & Outpatient & Mean 47.8 y & $\mathrm{MP}, \mathrm{CP}$ & 2003 & Serology \\
\hline $\begin{array}{l}\text { Miyashita et al, }{ }^{35} 2005 \\
\text { (Japan) }\end{array}$ & $\begin{array}{c}\text { Adults }>16 \text { y } \\
\text { with CAP }\end{array}$ & $506 / 318$ & $\begin{array}{l}400 \text { Inpatient, } \\
106 \text { outpatient }\end{array}$ & $\begin{array}{l}\text { Mean } 58.3 y \text {, } \\
\text { range } 16 y-97 y\end{array}$ & $M P, C P, L P$ & $1998-2003$ & Serology, UA \\
\hline $\begin{array}{l}\text { Molinos et al, }{ }^{36} 2009 \\
\text { (Spain) }\end{array}$ & $\begin{array}{l}\text { Patients } \\
\text { with CAPd }\end{array}$ & $710 / 274$ & Inpatient & Mean 67.1 y & $M P, C P, L P$ & $2003-2004$ & Serology, UA \\
\hline $\begin{array}{l}\text { Prat et al, }{ }^{37} 2006 \\
\text { (Spain) }\end{array}$ & $\begin{array}{l}\text { Patients } \\
\text { with CAPd }\end{array}$ & $217 / 116$ & Inpatient & Mean 56.6 y & LP & $2005-2005$ & UA \\
\hline $\begin{array}{l}\text { Saito et al, }{ }^{38} 2006 \\
\text { (Japan) }\end{array}$ & $\begin{array}{l}\text { Adults } \geq 17 \text { y } \\
\text { with CAP }\end{array}$ & $232 / 170$ & $\begin{array}{l}200 \text { Inpatient, } \\
32 \text { outpatient }\end{array}$ & $\begin{array}{l}\text { Mean } 60.2 y_{1} \\
\text { range } 17 y-99 y\end{array}$ & $M P, C P, L P$ & 1999-2000 & $\begin{array}{l}\text { Serology, } \\
\text { PCR, UA, } \\
\text { Culture }\end{array}$ \\
\hline $\begin{array}{l}\text { Sangil et al, }{ }^{39} 2012 \\
\text { (Spain) }\end{array}$ & $\begin{array}{l}\text { Adults } \geq 18 \text { y } \\
\text { with CAP }\end{array}$ & $131 / 92$ & Inpatient & $\begin{array}{l}\text { Mean } 64.4 y \\
\text { range } 48 y-80\end{array}$ & $M P, C P, L P$ & $2009-2010$ & $\begin{array}{l}\text { Serology, } \\
\text { PCR, UA }\end{array}$ \\
\hline $\begin{array}{l}\text { Shibli et al, }{ }^{40} 2010 \\
\text { (Israel) }\end{array}$ & $\begin{array}{l}\text { Adults } \geq 18 \text { y } \\
\text { with CAP }\end{array}$ & $126 / 84$ & Inpatient & $\begin{array}{l}\text { Mean } 58.3 \\
\text { range } 18 \text { y-93 y }\end{array}$ & $M P, C P, L P$ & $2006-2007$ & $\begin{array}{l}\text { Serology, } \\
\text { PCR }\end{array}$ \\
\hline $\begin{array}{l}\text { Stralin et al, }{ }_{, 1} 2010 \\
\text { (Sweden) }\end{array}$ & $\begin{array}{c}\text { Adults } \geq 18 \text { y } \\
\text { with CAP }\end{array}$ & $235 / 133$ & Inpatient & $\begin{array}{l}\text { Median } 71 y_{1} \\
\text { range } 18 y-96 \text { y }\end{array}$ & $M P, C P, L P$ & 1999-2002 & $\begin{array}{l}\text { Serology, } \\
P C R, U A\end{array}$ \\
\hline $\begin{array}{l}\text { Templeton et al,42 } \\
2005 \text { (Netherlands) }\end{array}$ & $\begin{array}{c}\text { Adults } \geq 18 \text { y } \\
\text { with CAP }\end{array}$ & $105 / 80$ & $\begin{array}{l}92 \text { inpatient, } \\
13 \text { outpatient }\end{array}$ & $\mathrm{NR}$ & $M P, C P, L P$ & $2000-2002$ & $P C R$ \\
\hline $\begin{array}{l}\text { van de Garde et al, }{ }^{43} \\
2008 \text { (Netherlands) }\end{array}$ & $\begin{array}{l}\text { Patients } \\
\text { with CAPd }\end{array}$ & $201 / 128$ & Inpatient & Mean 63 y & MP, LP & $2004-2006$ & PCR \\
\hline $\begin{array}{l}\text { von Baum et al, }{ }^{44} \\
2008 \text { (Germany } \\
\text { [CAPNETZ]) }\end{array}$ & $\begin{array}{l}\text { Adults } \geq 18 \text { y } \\
\text { with CAP }\end{array}$ & $2,503 / 877$ & $\begin{array}{l}\text { 1,727 Inpatient, } \\
776 \text { outpatient }\end{array}$ & Mean 61 y & LP & $2002-2005$ & $\begin{array}{l}\text { PCR, UA, } \\
\text { Culture }\end{array}$ \\
\hline $\begin{array}{l}\text { von Baum et al, }{ }^{45} \\
2009 \text { (Germany } \\
\text { [CAPNETZ]) }\end{array}$ & $\begin{array}{l}\text { Adults } \geq 18 \text { y } \\
\text { with CAP }\end{array}$ & $4,532 / 928$ & $\begin{array}{l}2,922 \text { Inpatient, } \\
\text { 1,610 outpatient }\end{array}$ & Mean $60 \mathrm{y}$ & $\mathrm{MP}$ & $2002-2005$ & $\begin{array}{l}\text { Serology, } \\
\text { PCR } \\
\text { continues }\end{array}$ \\
\hline \multicolumn{8}{|c|}{$\begin{array}{l}C A P=\text { community-acquired pneumonia; } C P=\text { Chlamydia pneumoniae; } L P=\text { Legionella pneumophila; } L R T I=\text { lower respiratory tract infection; } M P=\text { Mycoplasma pneumoniae; } \\
\mathrm{NR}=\text { not reported; } \mathrm{PCR}=\text { polymerase chain reaction; } \mathrm{PSI}=\text { pneumonia severity index; } \mathrm{UA}=\text { urine antigen testing. }\end{array}$} \\
\hline \multicolumn{8}{|c|}{$\begin{array}{l}\text { a Total = number of patients included in study. Confirmed = number of patients with a pathogen identified. } \\
\text { b Study findings reported separately for patients with CAP and those with non-pneumonic LRTI. } \\
\text { ' Estimated from median using method of Hozo. }{ }^{61} \\
{ }^{d} \text { Age not reported but presumably adult based on hospital and mean age. }\end{array}$} \\
\hline
\end{tabular}


Table 2. Characteristics of Studies of the Prevalence of Mycoplasma pneumoniae, Chlamydia pneumoniae, and Legionella pneumophila in Patients With Community-Acquired Pneumonia or Lower Respiratory Tract Infection (continued)

\begin{tabular}{|c|c|c|c|c|c|c|c|}
\hline $\begin{array}{l}\text { Author, Year } \\
\text { (Country) }\end{array}$ & Population & $\begin{array}{c}\text { Total I } \\
\text { Confirmed } \\
\text { Cases }^{a}\end{array}$ & Setting & Age & Pathogen & $\begin{array}{l}\text { Data } \\
\text { Collection } \\
\text { Period }\end{array}$ & $\begin{array}{l}\text { Diagnostic } \\
\text { Method }\end{array}$ \\
\hline \multicolumn{8}{|l|}{ CAP in Adults (continued) } \\
\hline $\begin{array}{l}\text { Wellinghausen et al, }{ }^{46} \\
2006 \text { (Germany } \\
\text { [CAPNETZ]) }\end{array}$ & $\begin{array}{c}\text { Adults } \geq 18 \text { y } \\
\text { with CAP }\end{array}$ & 546/NR & $\begin{array}{l}364 \text { Inpatient, } \\
182 \text { outpatient }\end{array}$ & Median 62 yi & $\mathrm{CP}$ & 2002-2004 & $P C R$ \\
\hline $\begin{array}{l}\text { Andreo et al, }{ }^{47} 2006 \\
\text { (Spain) }\end{array}$ & $\begin{array}{c}\text { Adults } \geq 16 \text { y } \\
\text { with CAP }\end{array}$ & $107 / 39$ & Inpatient & $\begin{array}{l}\text { Mean } 58.6 y, \\
\text { range } 16 y-86 y\end{array}$ & $M P, C P, L P$ & 2000-2001 & Serology \\
\hline $\begin{array}{l}\text { Capelastegui et al, }{ }^{48} \\
2012 \text { (Spain) }\end{array}$ & $\begin{array}{c}\text { Adults } \geq 18 \text { y } \\
\text { with CAP }\end{array}$ & $700 / 390$ & $\begin{array}{l}276 \text { Inpatient, } \\
424 \text { outpatient }\end{array}$ & Mean 59.7 y & $M P, C P, L P$ & 2006-2007 & Serology, UA \\
\hline \multicolumn{8}{|l|}{ CAP in Children } \\
\hline $\begin{array}{l}\text { Cantais et al, }{ }^{49} 2014 \\
\text { (France) }\end{array}$ & $\begin{array}{c}\text { Children age } \\
1 \text { mo to } 16.5 \mathrm{y} \\
\text { with CAP }\end{array}$ & $85 / 81$ & Inpatient & $\begin{array}{c}\text { Median } 2.8 \mathrm{y} \\
\text { range } 1 \mathrm{mo} \text { to } \\
16.5 \mathrm{y}\end{array}$ & $M P, C P$ & 2012-2013 & PCR \\
\hline $\begin{array}{l}\text { Cevey-Macherel et al, }{ }^{50} \\
2009 \text { (Switzerland) }\end{array}$ & $\begin{array}{l}\text { Children } 2 \text { mo to } \\
5 \text { y with CAP }\end{array}$ & $99 / 85$ & Inpatient & $\begin{array}{l}\text { Mean } 29 \text { mo, } \\
\text { range } 2 \text { mo to } 5 \text { y }\end{array}$ & $M P, C P$ & 2003-2005 & $\begin{array}{l}\text { Serology, } \\
\text { PCR }\end{array}$ \\
\hline $\begin{array}{l}\text { Don et al, } 2005 \\
\text { (Italy) }\end{array}$ & $\begin{array}{l}\text { Children } 4 \text { mo to } \\
16 \text { y with CAP }\end{array}$ & $101 / 66$ & Inpatient & $\begin{array}{l}\text { Mean } 4.7 y, \\
\text { range } 0.3 y-16 y\end{array}$ & $\mathrm{MP}, \mathrm{CP}$ & 2001-2002 & Serology \\
\hline $\begin{array}{l}\text { Hamano-Hasegawa } \\
\text { et al,52 } 2008 \text { (Japan) }\end{array}$ & $\begin{array}{l}\text { Children }<19 \text { y } \\
\text { with CAP }\end{array}$ & 1,700/1,316 & NR & $\begin{array}{c}\text { Median } 6.1 \text { y } \\
\text { for MP; Median } \\
5.4 \text { y for } C P \\
\text { Range } 0 \text { y-19 y }\end{array}$ & $M P, C P, L P$ & 2005-2006 & $P C R$ \\
\hline $\begin{array}{l}\text { Jain et al, }{ }^{53} 2015^{a} \\
\text { (United States) }\end{array}$ & $\begin{array}{c}\text { Children }<18 \text { y } \\
\text { with CAP }\end{array}$ & $2,222 / 1,802$ & Inpatient & $\begin{array}{l}\text { Median } 2 \text { y, } \\
\text { range } 0 y-17 y\end{array}$ & $\mathrm{MP}, \mathrm{CP}$ & $2010-2012$ & PCR \\
\hline $\begin{array}{l}\text { Kurz et al, }{ }^{54} 2013 \\
\quad \text { (Austria) }\end{array}$ & $\begin{array}{l}\text { Children } 2 \text { mo to } \\
17 \text { y with CAP }\end{array}$ & 279/190 & Inpatient & $\begin{array}{c}\text { Median } 36 \mathrm{mo} \text {, } \\
\text { range } 2 \mathrm{mo} \\
\text { to } 17 \mathrm{y}\end{array}$ & $M P, C P$ & 2005-2008 & $P C R$ \\
\hline $\begin{array}{l}\text { Laundy et al, }{ }^{55} 2003 \\
\quad \text { (England) }\end{array}$ & $\begin{array}{l}\text { Children }<5 \text { y } \\
\text { with CAP }\end{array}$ & $51 / 25$ & $\begin{array}{l}42 \text { Inpatient, } \\
9 \text { outpatient }\end{array}$ & $\begin{array}{c}\text { Median } 1.3 \mathrm{y} \\
\text { range } 2 \mathrm{wk} \\
\text { to } 4,8 \mathrm{y}\end{array}$ & $M P, C P$ & 2001-2002 & $P C R$ \\
\hline $\begin{array}{l}\text { Maltezou et al, }{ }^{56} \\
\text { 2004e (Greece) }\end{array}$ & $\begin{array}{l}\text { Children } 6 \text { mo } \\
\text { to } 14 \text { y with CAP } \\
\text { ( } n=60) \text {, cough } \\
>3 \text { weeks }(n=1) \\
\text { or infectious } \\
\text { asthma exacerba- } \\
\text { tion }(n=4)\end{array}$ & $65 / 19$ & Inpatient & $\begin{array}{l}\text { Mean } 6 y \text {, } \\
\text { range } 10 \mathrm{mo} \\
\text { to } 13 \mathrm{y}\end{array}$ & $M P, C P, L P$ & 2001 & Serology \\
\hline $\begin{array}{l}\text { Numazaki et al, }{ }^{57} \\
2004^{b} \text { (Japan) }\end{array}$ & $\begin{array}{l}\text { Children }<15 \text { y } \\
\text { with CAP }\end{array}$ & $398 / 383$ & $\begin{array}{l}362 \text { Inpatient, } \\
36 \text { outpatient }\end{array}$ & NR & $\mathrm{MP}, \mathrm{CP}$ & 2000-2001 & $\begin{array}{l}\text { Serology, } \\
\text { PCR }\end{array}$ \\
\hline $\begin{array}{l}\text { Tsolia et al, }{ }^{58} 2004 \\
\text { (Greece) }\end{array}$ & $\begin{array}{l}\text { Children } 5 y-14 \text { y } \\
\text { with CAP }\end{array}$ & $75 / 58$ & Inpatient & $\begin{array}{c}\text { Median } 86.5 \mathrm{mo}, \\
\text { range } 5 \mathrm{y}-14 \mathrm{y}\end{array}$ & $M P, C P$ & 2003 & $\begin{array}{l}\text { Serology, } \\
\text { PCR }\end{array}$ \\
\hline \multicolumn{8}{|l|}{ Nonpneumonia LRTI } \\
\hline $\begin{array}{l}\text { Graffelman et al, }{ }^{59} \\
2008^{f} \text { (Netherlands) }\end{array}$ & $\begin{array}{l}\text { Adults } \geq 18 \text { y con- } \\
\text { sulting GP with } \\
\text { LRTI; } 26 \text { of } 129 \\
\text { had CAP }\end{array}$ & $129 / 84$ & Outpatient & Mean 50 y & MP & 1998-2001 & $\begin{array}{l}\text { Serology, } \\
\text { PCR, } \\
\text { Culture }\end{array}$ \\
\hline $\begin{array}{l}\text { Numazaki et al, }{ }^{57} \\
2004^{b} \text { (Japan) }\end{array}$ & $\begin{array}{l}\text { Children }<15 \text { y } \\
\text { with non- } \\
\text { pneumonia LRTI }\end{array}$ & $523 / 470$ & $\begin{array}{l}436 \text { Inpatient, } \\
87 \text { outpatient }\end{array}$ & NR & $\mathrm{MP}, \mathrm{CP}$ & $2000-2001$ & $\begin{array}{l}\text { Serology, } \\
\text { PCR }\end{array}$ \\
\hline $\begin{array}{l}\text { Holm et al, }{ }^{29} 2007^{b} \\
\text { (Denmark) }\end{array}$ & $\begin{array}{c}\text { Adults } \geq 18 \text { y } \\
\text { with non- } \\
\text { pneumonia LRTI }\end{array}$ & $316 / 124$ & $\begin{array}{l}10 \text { Inpatient, } \\
306 \text { outpatient }\end{array}$ & $\begin{array}{l}\text { Median } 48 \text { y, } \\
\text { range } 18 \text { y-94 y }\end{array}$ & $\mathrm{MP}, \mathrm{CP}, \mathrm{LP}$ & 2002-2003 & $P C R$ \\
\hline \multicolumn{8}{|l|}{ Various } \\
\hline $\begin{array}{l}\text { Defilippi et al, }{ }^{60} 2008 \\
\text { (Italy) }\end{array}$ & $\begin{array}{l}\text { Children with LRTI } \\
\text { (acute bronchitis, } \\
\text { wheezy bronchi- } \\
\text { tis, pneumonia, } \\
\text { or bronchiolitis) } \\
\text { admitted to the } \\
\text { hospital }\end{array}$ & $886 / N R$ & & $\begin{array}{c}\text { Mean } 6.2 \mathrm{y} \\
\text { range } 1 \mathrm{mo} \\
\text { to } 13.5 \mathrm{y}\end{array}$ & MP & 2005-2006 & PCR \\
\hline \multicolumn{8}{|c|}{$\begin{array}{l}C A P=\text { community-acquired pneumonia; } C P=\text { Chlamydia pneumoniae; } L P=\text { Legionella pneumophila; } L R T I=\text { lower respiratory tract infection; } M P=\text { Mycoplasma pneumoniae; } \\
N R=\text { not reported; } P C R=\text { polymerase chain reaction; } P S I=\text { pneumonia severity index; } U A=\text { urine antigen testing. }\end{array}$} \\
\hline $\begin{array}{l}\text { a Total = number of patient } \\
\text { b Study findings reported se } \\
\text { ' Estimated from median us } \\
\text { d Age not reported but pres } \\
\text { e Classified as study of CAP }\end{array}$ & $\begin{array}{l}\text { ncluded in study. Confir } \\
\text { arately for patients with } \\
g \text { method of Hozo. }{ }^{61} \\
\text { mably adult based on ho } \\
\text { at least } 85 \% \text { of patients }\end{array}$ & $\begin{array}{l}\text { ed }=\text { number of } \\
\text { AP and those wit } \\
\text { pital and mean a } \\
\text { the series were }\end{array}$ & $\begin{array}{l}\text { tients with a pathoge } \\
\text { non-pneumonic LRTI }\end{array}$ & identified. & & & \\
\hline
\end{tabular}


unable to determine a reason for their high prevalence. There was also no clear pattern of variation by year of study.

Legionella pneumopbila was exceedingly rare in children, with only 1 case in 1,765 patients with CAP. ${ }^{52,56}$ The overall prevalence in adults was $2.8 \%$ (95\% CI, $2.1 \%-3.6 \%$ ), although in most studies it was between $1 \%$ and $3 \%$. Again, there was significant heterogeneity.
Of the studies reporting a prevalence of $5 \%$ or higher, 4 of 6 were in Spain, ${ }^{27,28,36,37}$ and a fifth, a study that also reported the highest prevalence of $\mathrm{C}$ pneumoniae, was set in another Mediterranean country, Israel. ${ }^{40}$ The largest series, set in Germany, found L pneumopbila in $3.7 \%$ of patients treated in ambulatory care and $3.8 \%$ of inpatients. ${ }^{44}$ Clearly, it is not only found in severely ill patients.

Figure 2. Forest plot of the prevalence of Mycoplasma pneumoniae in adults and children with communityacquired pneumonia, sorted in reverse chronological order.

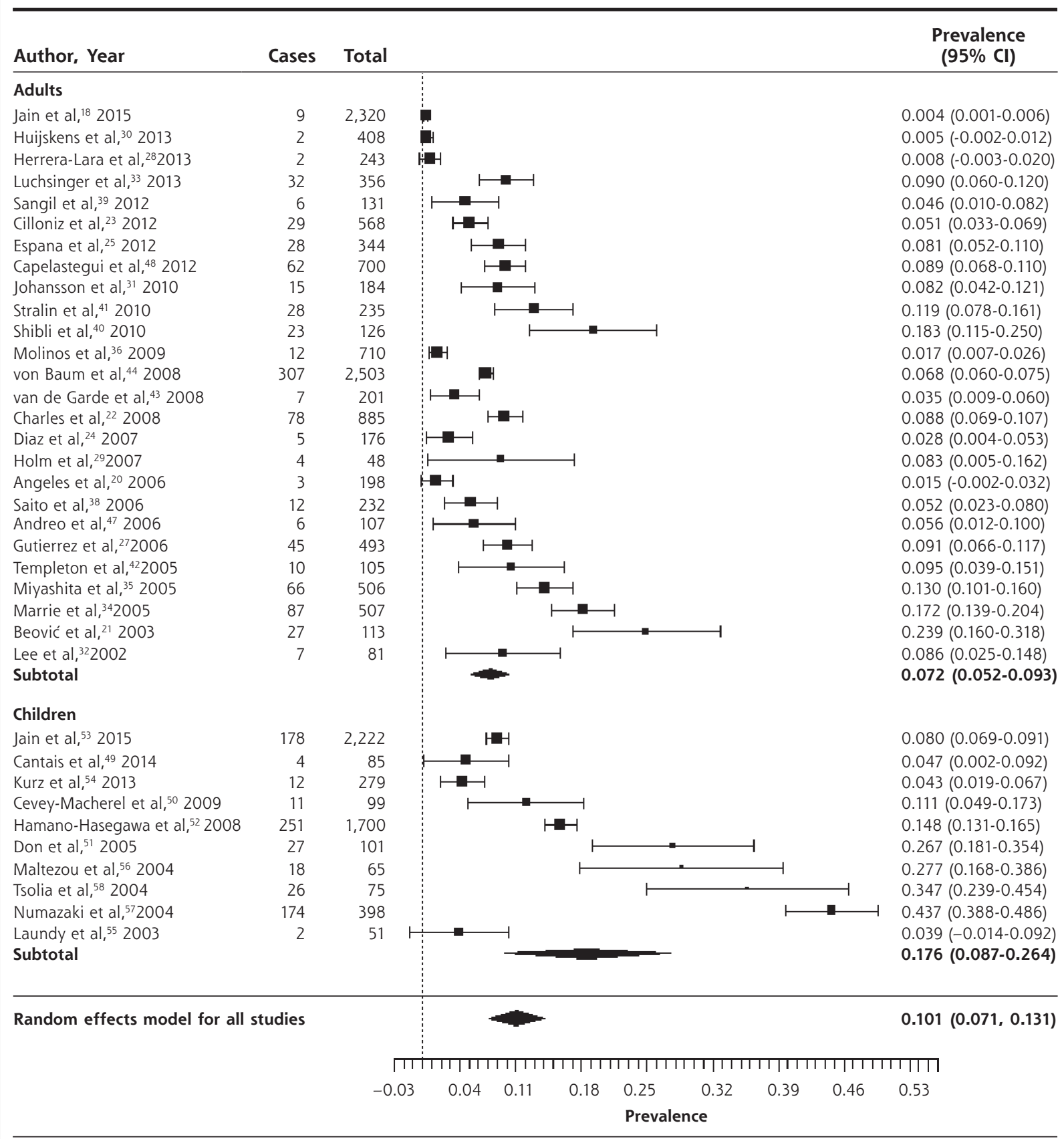

Heterogeneity $\left(\mathrm{I}^{2}\right)=99.27$ 


\section{Patients With Non-Pneumonia LRTI}

Two studies reported the prevalence of atypical pathogens in patients with LRTI in whom pneumonia had been excluded by normal chest radiography, ${ }^{29,57}$ and a third enrolled predominantly patients with nonpneumonia LRTI..$^{59}$ The prevalence of $M$ pneumoniae was $7 / 316(2.2 \%), 13 / 129(10.0 \%)$, and 78/523 (14.9\%) in these 3 studies, ${ }^{29,57,59}$ while the prevalence of $C$ pneumoniae was $2 / 316(0.6 \%)$ in 1 study $^{29}$ and $3 / 523(0.6 \%)$ in a second. ${ }^{57} \mathrm{~A}$ single study found no cases of $L$ pneumopbila in a primary care series of 316 adults with non-pneumonia LRTI. ${ }^{29} \mathrm{~A}$ fourth study did not provide adequate information to differentiate the number of children with acute bronchitis, pneumonia, or bronchiolitis. ${ }^{60}$

\section{Prevalence of Bordetella pertussis in Outpatients}

Table 3 summarizes data from 8 studies of the prevalence of $B$ pertussis in outpatients with prolonged or bothersome cough, largely in primary care. ${ }^{17,64-70}$ Three studies enrolled adults and children ${ }_{i}$, children only;

Figure 3. Forest plot of the prevalence of Chlamydia pneumoniae in adults and children with communityacquired pneumonia, sorted by prevalence.

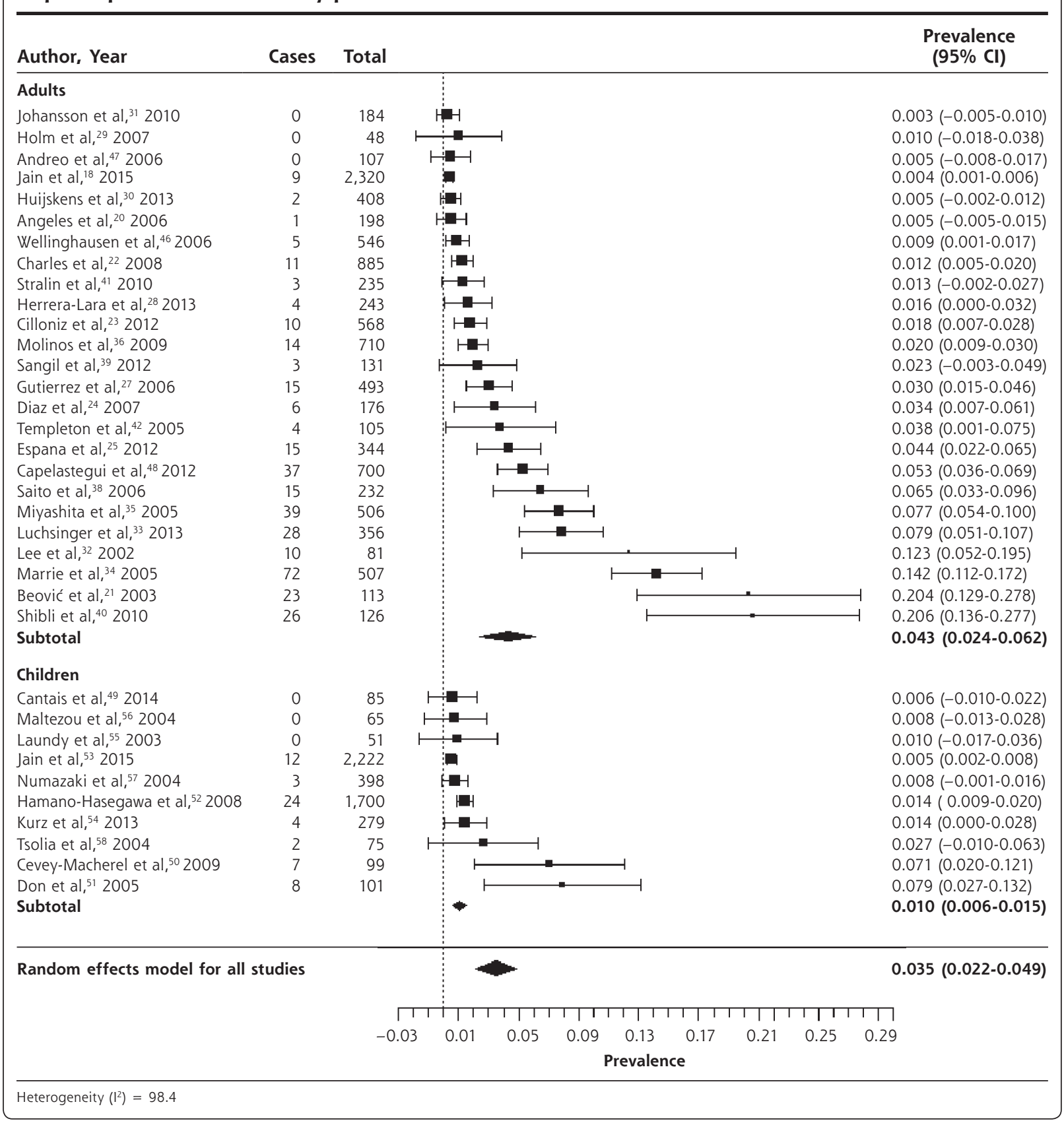


and 1, adults only. Data were collected between 2001 and 2012. One study assessed children referred from primary care due to suspicion for $B$ pertussis, based on the duration of cough. ${ }^{68}$ The prevalence of $B$ pertussis is summarized in the forest plot in Figure 5. While there was significant heterogeneity when including all studies, this was primarily due to heterogeneity in the 4 studies of children only.

Figure 4. Forest plot of the prevalence of Legionella pneumophila in adults and children with communityacquired pneumonia, sorted by prevalence.

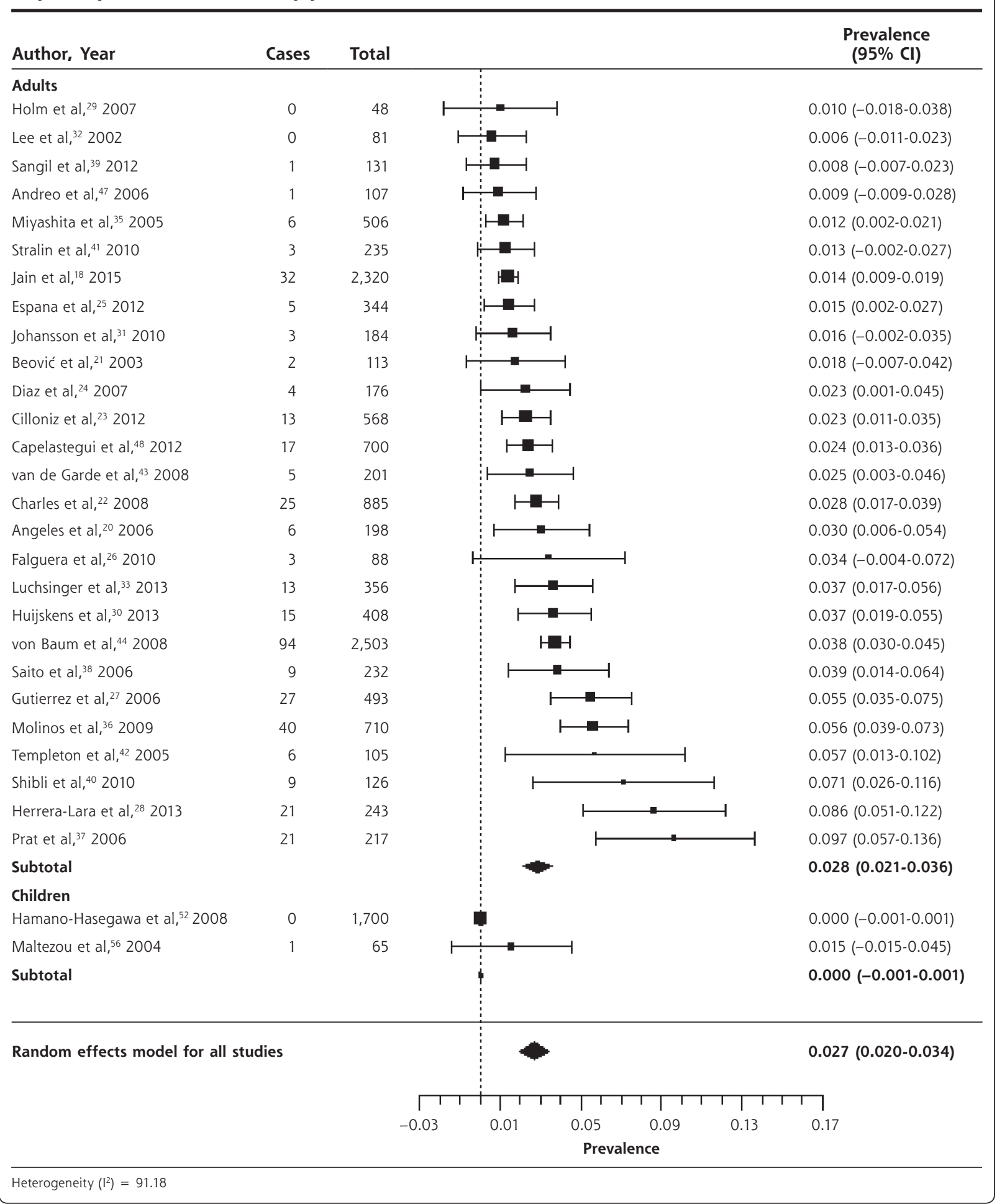


Table 3. Characteristics of Studies of the Prevalence of Bordetella pertussis in Outpatients With Prolonged Cough or Non-Pneumonia Lower Respiratory Tract Infection

\begin{tabular}{|c|c|c|c|c|}
\hline Author, Year & Population & Age & $\begin{array}{l}\text { Year of Data } \\
\text { Collection }\end{array}$ & $\begin{array}{l}\text { Diagnostic } \\
\text { Method }\end{array}$ \\
\hline \multicolumn{5}{|l|}{ Adults and children } \\
\hline Park et al, ${ }^{64} 2014$ (South Korea) & $\begin{array}{l}\text { Adolescents and adults age } 11 \text { y and older } \\
\text { presenting to GP with bothersome cough } \\
\text { up to } 30 \text { days duration }\end{array}$ & Mean 44.3 y & $2011-2012$ & PCR \\
\hline Philipson et al ${ }^{65} 2013$ (New Zealand) & $\begin{array}{l}\text { Children and adults age } 5 \text { to } 49 \text { y with } \\
\text { cough for } 2 \text { weeks or longer }\end{array}$ & Range $5-49$ y & 2011 & Serology \\
\hline Riffelmann et al, ${ }^{66} 2006$ (Germany) & $\begin{array}{l}\text { Patients presenting to GP with at least } \\
7 \text { days cough }\end{array}$ & $\begin{array}{l}\text { Not reported } \\
\text { (all ages) }\end{array}$ & $2001-2004$ & Serology or PCR \\
\hline \multicolumn{5}{|l|}{ Children } \\
\hline $\begin{array}{l}\text { Wang et al, }{ }^{67} 2014 \text { (United } \\
\text { Kingdom) }\end{array}$ & $\begin{array}{l}\text { Children with cough of 2-8 weeks duration } \\
\text { presenting to GP }\end{array}$ & Mean 9.6 y & $2010-2012$ & Serology \\
\hline $\begin{array}{l}\text { van den Brink et al, }{ }^{68} 2014 \\
\text { (Netherlands) }\end{array}$ & $\begin{array}{l}\text { Children age } 12 \text { y and under with RTI } \\
\text { referred for evaluation of suspected BP }\end{array}$ & $<12 y$ & 2007-2009 & PCR \\
\hline $\begin{array}{l}\text { Harnden et al, }{ }^{69} 2006 \text { (England, } \\
\text { United Kingdom) }\end{array}$ & $\begin{array}{l}\text { Children } 5-16 \text { y presenting to their GP with } \\
\text { cough for at least } 2 \text { weeks }\end{array}$ & $\begin{array}{l}\text { Mean age } 9.4 y_{1} \\
\text { range } 5-17\end{array}$ & $2001-2005$ & Serology \\
\hline Diez Domingo et al, ${ }^{70} 2004$ (Spain) & $\begin{array}{l}\text { Children age } 15 \mathrm{y} \text { and under presenting } \\
\text { with cough for at least } 2 \text { weeks }\end{array}$ & $\begin{array}{l}\text { Mean } 6.2 y_{1} \\
\text { range } 0-15 y\end{array}$ & $2001-2002$ & Serology \\
\hline \multicolumn{5}{|c|}{ 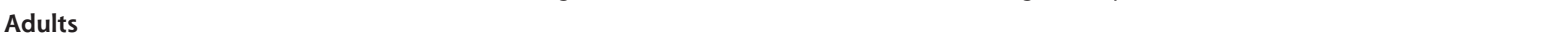 } \\
\hline $\begin{array}{l}\text { Teepe et al, }{ }^{17} 2015 \text { (12 European } \\
\text { countries) }\end{array}$ & $\begin{array}{l}\text { Adults with acute cough }<28 \text { days duration } \\
\text { presenting to GP }\end{array}$ & Mean age $50 y$ & $2007-2010$ & Serology or $\mathrm{PCR}$ \\
\hline
\end{tabular}

The overall prevalence was $12.4 \%$ (95\% CI, $4.9 \%$ $19.8 \%)$. In a large, multi-country, European prospective study of adults presenting to primary care with cough of up to 28 days duration, ${ }^{17}$ prevalence was $3 \%(95 \%$ $\mathrm{CI}, 2.4 \%-3.6 \%)$. The prevalence was higher in studies of children $(17.6 \% ; 95 \% \mathrm{CI}, 3.4 \%-31.8 \%)$ than in those of adults and children $(8.9 \% ; 95 \% \mathrm{CI}, 6.7 \%-11.2 \%)$, but there was significant heterogeneity in the studies of children, with a range from $4.6 \%$ to $37.2 \% .67-70$

\section{Surveillance Data for Bordetella pertussis and Legionella pneumophila}

Of the 26 countries to report data on B pertussis, Australia had the highest incidence rate of 105.0 cases per 100,000 persons per year. Hungary reported the lowest incidence rate of 0.05 cases per 100,000 persons per year. With 48,277 cases, the United States had the most reported cases of all countries, twice as many as the next country. Of the 30 countries reporting $L$ pneumopbila, the United States had the most cases at 3,688. Poland reported the lowest incidence of $L$ pneumopbila (0.02 per 100,000 persons per year) and Slovenia the highest (4.02 per 100,000 persons per year). It is likely that differences in surveillance systems and reporting account for much of this variability.

\section{DISCUSSION}

Among adults with CAP, $14 \%$ had an atypical pathogen: $7 \%$ had Mycoplasma pneumoniae, $4 \%$ had Chla- mydopbila pneumoniae, and 3\% had Legionella pneumopbila. Among children with CAP, $18 \%$ had Mycoplasma pneumoniae, only $1 \%$ had Chlamydopbila pneumoniae, and Legionella pneumopbila was extremely rare (1 case in 1,765 patients). Among patients with prolonged cough, 9\% of adults and $18 \%$ of children had Bordetella pertussis.

\section{Evidence for Underdiagnosis}

CAP is diagnosed in an estimated 5.6 million patients annually in the United States, and 1.1 million hospitalizations result. ${ }^{71,72}$ Laboratory-based surveillance, however, identifies only 3,700 infections caused by $L$ pneumophila each year, or $0.06 \%$ of all community-acquired pneumonias. Our systematic review found that when a consecutive series of patients with CAP are all tested for L pneumopbila, it is detected in $3 \%$ of patients, with a range of $1 \%$ to $10 \%$. This is consistent with the most recent US study, ${ }^{18}$ which found that $1.9 \%$ of episodes of CAP in a consecutive series of hospitalized adults were caused by $L$ pneumophila. If $2 \%$ of all episodes of CAP are caused by L pneumophila, this would be 112,000 cases per year. Thus, the vast majority of cases of $L$ pneumopbila in the United States, approximately 100,000 , may be undiagnosed. It is therefore important that physicians consider this pathogen when diagnosing $\mathrm{CAP}$, and consider ordering urine antigen tests for L pneumopbila more routinely, particularly when patients are non-responsive or slowly responsive to therapy with a beta-lactam. The recommended antibiotic for L pneumopbila is a respiratory fluoroquinolone. ${ }^{73,74}$ 
Similarly, the annual incidence of acute bronchitis or non-pneumonia LRTI is approximately 440 episodes in 10,000 adults, $^{75}$ and the annual incidence of $B$ pertussis based on surveillance is 1.5 of 10,000 persons. Our systematic review found that $18 \%$ of episodes of nonpneumonia LRTI in children and $9 \%$ of those in adults were caused by $B$ pertussis. Most of these studies limited inclusion to patients with a cough for at least 1 to 2 weeks, although 1 included adults and children with a shorter duration of cough and still found a prevalence of $7 \%{ }^{64}$ If one conservatively estimates based on these data that $3 \%$ of episodes of acute bronchitis or non-pneumonia LRTI are caused by $B$ pertussis, that corresponds to 13 episodes per 10,000. Again, these data suggest that there is widespread underdiagnosis of $B$ pertussis in the United States, with approximately $90 \%$ of episodes undiagnosed. This is important because family members and relatives are the source for $75 \%$ to $83 \%$ of pertussis cases in infants. ${ }^{76,77}$ Moreover, immunization with the pertussis vaccine wanes after five years. ${ }^{78-80}$ Current recommendations to vaccinate pregnant women with Tdap should be closely adhered to.

C pneumoniae infection has traditionally been described as being more common in children. We found that the mean prevalence, however, was $4 \%$ in studies of adults with CAP compared with $1 \%$ in children.

Diagnosis of these infections could be improved in several ways. One is to make better use of the history

Figure 5. Forest plot of the prevalence of Bordetella pertussis in outpatients with prolonged cough or non-pneumonia lower respiratory tract infection, sorted by prevalence.

\begin{tabular}{|c|c|c|c|c|c|}
\hline Author, Year & $\begin{array}{l}\text { Cough } \\
\text { Duration }\end{array}$ & Cases & Total & & $\begin{array}{c}\text { Prevalence } \\
(95 \% \mathrm{Cl})\end{array}$ \\
\hline \multicolumn{6}{|l|}{ Adults } \\
\hline Teepe et al, ${ }_{1}^{17} 2015$ & $\leq 28$ days & 93 & 3,074 & $\square$ & $0.030(0.024-0.036)$ \\
\hline Subtotal & & & & + & $0.030(0.024-0.036)$ \\
\hline \multicolumn{6}{|l|}{ Adults and Children } \\
\hline Park et al, ${ }^{64} 2014$ & $\leq 30$ days & 34 & 490 & $\mathrm{H}$ & $0.069(0.047-0.092)$ \\
\hline Riffelmann et al, ${ }^{66} 2006$ & $\geq 7$ days & 97 & 971 & $\mapsto H$ & $0.100(0.081-0.119)$ \\
\hline Philipson et al, ${ }^{65} 2013$ & $>14$ days & 23 & 222 & $\mapsto-1$ & $0.104(0.064-0.144)$ \\
\hline Subtotal & & & & 4 & $0.089(0.067-0.112)$ \\
\hline \multicolumn{6}{|l|}{ Children } \\
\hline van den Brink et al, ${ }^{68} 2014$ & Not reported & 14 & 306 & $\mathrm{H}$ & $0.046(0.022-0.069)$ \\
\hline Diez Domingo et al, ${ }^{70} 2004$ & $>14$ days & 5 & 57 & $\longmapsto$ & $0.088(0.014-0.161)$ \\
\hline Wang et al, ${ }^{67} 2014$ & $>14$ days & 56 & 273 & $\longmapsto-1$ & $0.205(0.157-0.253)$ \\
\hline Harnden et al, ${ }^{69} 2006$ & $>14$ days & 64 & 172 & $\longmapsto-1$ & $0.372(0.300-0.444)$ \\
\hline \multicolumn{5}{|l|}{ Subtotal } & $0.176(0.034-0.318)$ \\
\hline \multirow{3}{*}{\multicolumn{2}{|c|}{ Random effects model for all studies }} & & & & $0.124(0.049-0.198)$ \\
\hline & & & & 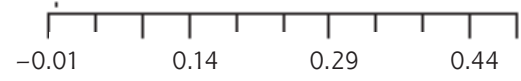 & \\
\hline & & \multicolumn{4}{|c|}{ Prevalence } \\
\hline
\end{tabular}


and physical examination. The best evidence regarding diagnosis of each pathogen is summarized in Table 4.

Data regarding diagnosis are quite limited, and only in

Table 4. Accuracy of Signs and Symptoms for Respiratory Infections With Atypical Pathogens

\begin{tabular}{|c|c|c|c|c|}
\hline $\begin{array}{l}\text { Symptom or Sign } \\
\text { (number of studies) }\end{array}$ & $\begin{array}{l}\text { Sensitivity } \\
(95 \% \mathrm{Cl})\end{array}$ & $\begin{array}{l}\text { Specificity } \\
(95 \% \mathrm{Cl})\end{array}$ & $\begin{array}{l}\text { Positive LR } \\
(95 \% \mathrm{Cl})\end{array}$ & $\begin{array}{c}\text { Negative LR } \\
(95 \% \mathrm{Cl})\end{array}$ \\
\hline \multicolumn{5}{|l|}{ Mycoplasma pneumoniae ${ }^{\mathrm{a}}$} \\
\hline Cough (5) & $\begin{array}{l}0.89 \\
(0.67-0.97)\end{array}$ & $\begin{array}{l}0.15 \\
(0.05-0.37)\end{array}$ & $\begin{array}{l}1.04 \\
(0.95-1.13)\end{array}$ & $\begin{array}{l}0.78 \\
(0.44-1.39)\end{array}$ \\
\hline Wheeze (6) & $\begin{array}{l}0.25 \\
(0.17-0.36)\end{array}$ & $\begin{array}{l}0.67 \\
(0.56-0.76)\end{array}$ & $\begin{array}{l}0.76 \\
(0.60-0.97)\end{array}$ & $\begin{array}{l}1.12 \\
(1.02-1.23)\end{array}$ \\
\hline Coryza (4) & $\begin{array}{l}0.32 \\
(0.08-0.72)\end{array}$ & $\begin{array}{l}0.66 \\
(0.28-0.91)\end{array}$ & $\begin{array}{l}0.95 \\
(0.71-1.26)\end{array}$ & $\begin{array}{l}1.03 \\
(0.90-1.17)\end{array}$ \\
\hline Crepitations (5) & $\begin{array}{l}0.84 \\
(0.78-0.88)\end{array}$ & $\begin{array}{l}0.22 \\
(0.14-0.32)\end{array}$ & $\begin{array}{l}1.06 \\
(0.96-1.18)\end{array}$ & $\begin{array}{l}0.77 \\
(0.52-1.12)\end{array}$ \\
\hline Fever (5) & $0.53-0.94$ & $0.02-0.43$ & & \\
\hline Rhonchi (4) & $0.11-0.74$ & $0.33-0.81$ & & \\
\hline Chest pain (2) & $0.08-0.19$ & $0.93-0.97$ & & \\
\hline Diarrhea (2) & $0.14-0.21$ & $0.79-0.85$ & & \\
\hline \multicolumn{5}{|l|}{ Chlamydophila pneumoniae } \\
\hline \multicolumn{5}{|l|}{ Adults $^{b}$} \\
\hline History of cough & 0.81 & & & \\
\hline History of sore throat & 0.52 & & & \\
\hline Abnormal breathing sounds & 0.38 & & & \\
\hline History of fever & 0.24 & & & \\
\hline \multicolumn{5}{|l|}{ Childrenc } \\
\hline Rales & 0.85 & & & \\
\hline Fever & 0.80 & & & \\
\hline Cough & 0.50 & & & \\
\hline Rhinitis & 0.30 & & & \\
\hline Tachypnea & 0.25 & & & \\
\hline Wheezes & 0.20 & & & \\
\hline Rhonchi & 0.15 & & & \\
\hline Legionella pneumophila & aOR $(95 \% \mathrm{Cl})$ & & & \\
\hline C-reactive protein $>187 \mathrm{mg}, \mathrm{L}$ & $4.4(2.0-9.6)$ & & & \\
\hline Sodium <133 mmo/L & $4.5(2.2-9.0)$ & & & \\
\hline Temperature $>39.4^{\circ} \mathrm{C}$ & $4.3(1.9-9.8)$ & & & \\
\hline Platelet count $<171 \times 10^{3} / \mathrm{mL}$ & $1.2(0.6-2.5)$ & & & \\
\hline $\begin{array}{l}\text { Lactate dehydrogenase } \\
>225 \mathrm{mmol} / \mathrm{L}\end{array}$ & $1.7(0.4-7.6)$ & & & \\
\hline Dry cough & $0.6(0.3-1.4)$ & & & \\
\hline \multicolumn{5}{|l|}{ Bordetella pertussis } \\
\hline Paroxysmal cough & & & $1.1(1.1-1.2)$ & $\begin{array}{l}0.52 \\
(0.27-.0)\end{array}$ \\
\hline Posttussive emesis & & & $1.8(1.4-2.2)$ & $\begin{array}{l}0.58 \\
(0.44-0.77)\end{array}$ \\
\hline Inspiratory whoop & & & $1.9(1.4-2.6)$ & $\begin{array}{l}0.78 \\
(0.66-0.93)\end{array}$ \\
\hline \multicolumn{5}{|c|}{$\mathrm{aOR}=$ adjusted odds ratio from multivariate analysis; $C A P=$ community-acquired pneumonia; $\mathrm{LR}=$ likelihood ratio. } \\
\hline \multicolumn{5}{|c|}{$\begin{array}{l}\text { a Cochrane systematic review of } 7 \text { moderate quality studies with a total of } 1,491 \text { children, although each sign and } \\
\text { symptom was only reported by a subset of studies. Pooled results from } 4 \text { to } 6 \text { studies are shown for cough, wheeze, } \\
\text { coryza, and crepitations; for the other signs and symptoms, a range or the results of a single study are shown. }{ }^{81}\end{array}$} \\
\hline \multicolumn{5}{|c|}{$\begin{array}{l}{ }^{b} \text { Data from a study of } 21 \text { adult primary care patients diagnosed with Chlamydophila pneumoniae infection ( } 7 \text { pri- } \\
\text { mary infections and } 14 \text { with reinfection based on the antibody pattern). }{ }^{82}\end{array}$} \\
\hline \multicolumn{5}{|c|}{ ' Data from a study of 20 children hospitalized for CAP and diagnosed with Chlamydophila pneumoniae. ${ }^{83}$} \\
\hline \multicolumn{5}{|c|}{$\begin{array}{l}\text { d Data from } 37 \text { patients hospitalized with CAP due to Legionella pneumophila. A clinical rule that included } 6 \text { variables } \\
\text { had an area under the receiver operating curve of } 0.73 .^{84}\end{array}$} \\
\hline \multicolumn{5}{|c|}{$\begin{array}{l}\text { e Systematic review of } 3 \text { studies with a total of } 486 \text { adults and children set in South Korea, United Kingdom, and } \\
\text { United States. } .^{85}\end{array}$} \\
\hline
\end{tabular}

the case of L pneumophila has an attempt been made to develop and validate a clinical decision rule that combines several signs and symptoms. ${ }^{84}$ In general, individual signs and symptoms are of little value in the diagnosis of these atypical pathogens. Another approach would be to integrate signs and symptoms with a point-of-care test such as c-reactive protein (CRP), as has been done for pneumonia and influenza diagnosis. ${ }^{86,87}$ Greater use of urine antigen tests for L pneumopbila should be encouraged for patients diagnosed with CAP, and the development of accurate, rapid point-of-care tests for $C$ pneumoniae and $B$ pertussis should be prioritized.

\section{Limitations}

As with any systematic review, our conclusions are limited by the quality of the published literature and the completeness and accuracy of reporting. We found considerable heterogeneity. For M pneumoniae this may be related to the cyclical nature of outbreaks, while for other pathogens the cause is less clear but may may lie in the differences in the populations studied, varying laboratory techniques, and varying sample collection methods across countries. It is noteworthy that the majority of studies found similar prevalences, with the heterogeneity for $C$ pneumoniae and L pneumopbila introduced by a small number of outliers, and for $B$ pertussis limited to studies in children only. We limited our analysis to studies that gathered data within the past 15 years in highly developed economies, so our findings may not be generalizable to low- or middle-income countries. Many patients with acute cough do not seek care. 
It is possible that those seeking care have a different (and perhaps more severe) illness and a different prevalence of these pathogens. Finally, the literature regarding the prevalence of pathogens in patients with non-pneumonia lower respiratory tract infection is quite limited, with no studies in the United States or Canada.

We have demonstrated that atypical bacterial pathogens are relatively common causes of CAP in a range of populations including both adults and children, and that $B$ pertussis is a common cause of prolonged cough. We do not feel that broader use of antibiotics for patients with acute cough is warranted. What is needed are studies to help clinicians more accurately diagnose these pathogens or to help them identify a large group of patients at low risk for such pathogens who do not require further testing or antibiotic therapy. Approaches that develop clinical decision rules integrating signs, symptoms, and point-of-care tests such as CRP are particularly promising. ${ }^{88}$ Finally, research is needed to determine if and when antibiotics are helpful, since data regarding treatment of $B$ pertussis and $M$ pneumoniae from well designed, adequately powered contemporary clinical trials are lacking.

To read or post commentaries in response to this article, see it online at http://www.annfammed.org/content/14/6/552.

Key words: community acquired pneumonia; cough; respiratory tract infection; Mycoplasma pneumoniae; Chlamydophila pneumoniae; Legionella pneumophila; Bordetella pertussis

Submitted February 5, 2016; submitted, revised, June 2, 2016; accepted July 13, 2016.

Supplementary materials: Available at http://www.AnnFamMed. org/content/14/6/552/suppl/DC1/

\section{References}

1. Hsiao CJ, Cherry D, Beatty PC, Rechtsteiner EA. National Ambulatory Medical Care Survey: 2007 summary. Hyattsville, MD: National Center for Health Statistics; 2010. National Health Statistics Reports, No. 27.

2. van Vugt SF, Verheij TJ, de Jong PA, et al; GRACE Project Group. Diagnosing pneumonia in patients with acute cough: clinical judgment compared to chest radiography. Eur Respir J. 2013;42(4):1076-1082.

3. Centers for Disease Control and Prevention. 2014 Provisional Pertussis Surveillance Report. http://www.cdc.gov/pertussis/downloads/ pertuss-surv-report-2014.pdf. Published Oct 2015.

4. Omori R, Nakata Y, Tessmer HL, Suzuki S, Shibayama K. The determinant of periodicity in Mycoplasma pneumoniae incidence: an insight from mathematical modelling. Sci Rep. 2015;5:14473.

5. Nguipdop-Djomo P, Fine P, Halsby K, Chalker V, Vynnycky E. Cyclic epidemics of Mycoplasma pneumoniae infections in England and Wales from 1975 to 2009: time-series analysis and mathematical modelling. The Lancet. 2013;382(S78).

6. Centers for Disease Control and Prevention (CDC). Mycoplasma pneumoniae outbreak at a university - Georgia, 2012. MMWR Morb Mortal Wkly Rep. 2013;62(30):603-606.

7. Zhan P, Suo LJ, Qian Q, et al. Chlamydia pneumoniae infection and lung cancer risk: a meta-analysis. Eur J Cancer. 2011;47(5):742-747.
8. Hahn DL, Schure A, Patel K, Childs T, Drizik E, Webley W. Chlamydia pneumoniae-specific IgE is prevalent in asthma and is associated with disease severity. PLoS One. 2012;7(4):e35945.

9. Johnston SL, Martin RJ. Chlamydophila pneumoniae and Mycoplasma pneumoniae: a role in asthma pathogenesis? Am J Respir Crit Care Med. 2005;172(9):1078-1089.

10. Phin N, Parry-Ford F, Harrison T, et al. Epidemiology and clinical management of Legionnaires' disease. Lancet Infect Dis. 2014;14(10): 1011-1021.

11. Fisman DN, Lim S, Wellenius GA, et al. It's not the heat, it's the humidity: wet weather increases legionellosis risk in the greater Philadelphia metropolitan area. J Infect Dis. 2005;192(12):2066-2073.

12. Shaikh R, Guris D, Strebel PM, Wharton M. Underreporting of pertussis deaths in the United States: need for improved surveillance. Pediatrics. 1998;101(2):323.

13. Organisation for Economic Co-operation and Development (OECD). List of high income OECD countries and high income Euro area countries. In: Country Classification 2011 - as of 26 July 2011. https:// www.oecd.org/tad/xcred/48405330.pdf. Accessed Oct 31, 2016.

14. The Cochrane Collaboration. Assessing risk of bias in included studies. In: Higgins J, Green S, eds. Cochrane Handbook for Systematic Reviews of Interventions. http://handbook.cochrane.org/chapter_8/8_assessing_risk_of_bias_in_included_studies.htm. Updated Mar 2011.

15. Whiting PF, Rutjes AW, Westwood ME, et al; QUADAS-2 Group. QUADAS-2: a revised tool for the quality assessment of diagnostic accuracy studies. Ann Intern Med. 2011;155(8):529-536.

16. Shamliyan T, Kane RL, Dickinson S. A systematic review of tools used to assess the quality of observational studies that examine incidence or prevalence and risk factors for diseases. J Clin Epidemiol. 2010;63(10):1061-1070.

17. Teepe J, Broekhuizen BD, leven M, et al; GRACE consortium. Prevalence, diagnosis, and disease course of pertussis in adults with acute cough: a prospective, observational study in primary care. $\mathrm{Br} J \mathrm{Gen}$ Pract. 2015;65(639):e662-e667.

18. Jain S, Self WH, Wunderink RG, et al; CDC EPIC Study Team. Community-acquired pneumonia requiring hospitalization among U.S. adults. N Engl J Med. 2015;373(5):415-427.

19. Gapminder. Population, total. In: Data in Gapminder World. http:// www.gapminder.org/data. Accessed June 2015.

20. Angeles Marcos M, Camps M, Pumarola T, et al. The role of viruses in the aetiology of community-acquired pneumonia in adults. Antivir Ther. 2006;11(3):351-359.

21. Beović B, Bonac B, Kese D, et al. Aetiology and clinical presentation of mild community-acquired bacterial pneumonia. Eur J Clin Microbiol Infect Dis. 2003;22(10):584-591.

22. Charles PG, Whitby M, Fuller AJ, et al; Australian CAP Study Collaboration. The etiology of community-acquired pneumonia in Australia: why penicillin plus doxycycline or a macrolide is the most appropriate therapy. Clin Infect Dis. 2008;46(10):1513-1521.

23. Cillóniz C, Ewig S, Polverino E, et al. Community-acquired pneumonia in outpatients: aetiology and outcomes. Eur Respir J. 2012;40(4): 931-938.

24. Díaz A, Barria P, Niederman M, et al. Etiology of communityacquired pneumonia in hospitalized patients in chile: the increasing prevalence of respiratory viruses among classic pathogens. Chest. 2007;131(3):779-787.

25. España PP, Capelastegui A, Bilbao A, et al; Population Study of Pneumonia (PSOP) Group. Utility of two biomarkers for directing care among patients with non-severe community-acquired pneumonia. Eur J Clin Microbiol Infect Dis. 2012;31(12):3397-3405.

26. Falguera M, Ruiz-González A, Schoenenberger JA, et al. Prospective, randomised study to compare empirical treatment versus targeted treatment on the basis of the urine antigen results in hospitalised patients with community-acquired pneumonia. Thorax. 2010;65(2):101-106. 
27. Gutiérrez F, Masiá M, Mirete C, et al. The influence of age and gender on the population-based incidence of community-acquired pneumonia caused by different microbial pathogens. J Infect. 2006; 53(3):166-174.

28. Herrera-Lara S, Fernández-Fabrellas E, Cervera-Juan Á, Blanquer-Olivas $R$. Do seasonal changes and climate influence the etiology of community acquired pneumonia? Arch Bronconeumol. 2013;49(4):140-145.

29. Holm A, Nexoe J, Bistrup LA, et al. Aetiology and prediction of pneumonia in lower respiratory tract infection in primary care. $\mathrm{Br}$ J Gen Pract. 2007;57(540):547-554.

30. Huijskens EG, van Erkel AJ, Palmen FM, Buiting AG, Kluytmans JA Rossen JW. Viral and bacterial aetiology of community-acquired pneumonia in adults. Influenza Other Respir Viruses. 2013;7(4):567-573.

31. Johansson N, Kalin M, Tiveljung-Lindell A, Giske CG, Hedlund J. Etiology of community-acquired pneumonia: increased microbiological yield with new diagnostic methods. Clin Infect Dis. 2010;50 (2):202-209

32. Lee SJ, Lee MG, Jeon MJ, Jung KS, Lee HK, Kishimoto T. Atypical pathogens in adult patients admitted with community-acquired pneumonia in Korea. Jpn J Infect Dis. 2002;55(5):157-159.

33. Luchsinger V, Ruiz M, Zunino $E$, et al. Community-acquired pneumonia in Chile: the clinical relevance in the detection of viruses and atypical bacteria. Thorax. 2013;68(11):1000-1006.

34. Marrie TJ, Poulin-Costello M, Beecroft MD, Herman-Gnjidic Z. Etiology of community-acquired pneumonia treated in an ambulatory setting. Respir Med. 2005;99(1):60-65.

35. Miyashita N, Fukano H, Mouri K, et al. Community-acquired pneumonia in Japan: a prospective ambulatory and hospitalized patient study. J Med Microbiol. 2005;54(Pt 4):395-400.

36. Molinos L, Clemente MG, Miranda B, et al; ASTURPAR Group. Community-acquired pneumonia in patients with and without chronic obstructive pulmonary disease. J Infect. 2009;58(6):417-424.

37. Prat C, Domínguez J, Andreo F, et al. Procalcitonin and neopterin correlation with aetiology and severity of pneumonia. J Infect. 2006;52(3):169-177.

38. Saito A, Kohno S, Matsushima T, et al; Study Group. Prospective multicenter study of the causative organisms of community-acquired pneumonia in adults in Japan. J Infect Chemother. 2006;12(2):63-69.

39. Sangil A, Calbo E, Robles A, et al. Aetiology of communityacquired pneumonia among adults in an H1N1 pandemic year: the role of respiratory viruses. Eur J Clin Microbiol Infect Dis. 2012;31(10): 2765-2772.

40. Shibli F, Chazan B, Nitzan O, et al. Etiology of community-acquired pneumonia in hospitalized patients in northern Israel. Isr Med Assoc J. 2010;12(8):477-482.

41. Strålin $K$, Olcén $P$, Törnqvist $E$, Holmberg $H$. Definite, probable, and possible bacterial aetiologies of community-acquired pneumonia at different CRB-65 scores. Scand J Infect Dis. 2010;42(6-7):426-434.

42. Templeton KE, Scheltinga SA, van den Eeden WC, Graffelman AW, van den Broek PJ, Claas EC. Improved diagnosis of the etiology of community-acquired pneumonia with real-time polymerase chain reaction. Clin Infect Dis. 2005;41(3):345-351.

43. van de Garde EM, Endeman H, van Hemert RN, et al. Prior outpatient antibiotic use as predictor for microbial aetiology of community-acquired pneumonia: hospital-based study. Eur J Clin Pharmacol. 2008;64(4):405-410.

44. von Baum H, Ewig S, Marre R, et al; Competence Network for Community Acquired Pneumonia Study Group. Community-acquired Legionella pneumonia: new insights from the German competence network for community acquired pneumonia. Clin Infect Dis. 2008:46(9):1356-1364.

45. von Baum H, Welte T, Marre R, Suttorp N, Lück C, Ewig S. Mycoplasma pneumoniae pneumonia revisited within the German Competence Network for Community-acquired pneumonia (CAPNETZ). BMC Infect Dis. 2009;9:62.
46. Wellinghausen $N$, Straube E, Freidank $H$, von Baum H, Marre R, Essig A. Low prevalence of Chlamydia pneumoniae in adults with community-acquired pneumonia. Int J Med Microbiol. 2006;296(7): 485-491.

47. Andreo F, Domínguez J, Ruiz J, et al. Impact of rapid urine antigen tests to determine the etiology of community-acquired pneumonia in adults. Respir Med. 2006;100(5):884-891.

48. Capelastegui A, España PP, Bilbao A, et al; Poblational Study of Pneumonia (PSoP) Group. Etiology of community-acquired pneumonia in a population-based study: link between etiology and patients characteristics, process-of-care, clinical evolution and outcomes. BMC Infect Dis. 2012;12:134.

49. Cantais A, Mory O, Pillet S, et al. Epidemiology and microbiological investigations of community-acquired pneumonia in children admitted at the emergency department of a university hospital. J Clin Virol. 2014;60(4):402-407.

50. Cevey-Macherel M, Galetto-Lacour A, Gervaix A, et al. Etiology of community-acquired pneumonia in hospitalized children based on WHO clinical guidelines. Eur J Pediatr. 2009;168(12):1429-1436.

51. Don M, Fasoli L, Paldanius M, et al. Aetiology of communityacquired pneumonia: serological results of a paediatric survey. Scand J Infect Dis. 2005;37(11-12):806-812.

52. Hamano-Hasegawa K, Morozumi M, Nakayama E, et al; Acute Respiratory Diseases Study Group. Comprehensive detection of causative pathogens using real-time $P C R$ to diagnose pediatric communityacquired pneumonia. J Infect Chemother. 2008;14(6):424-432.

53. Jain S, Williams DJ, Arnold SR, et al; CDC EPIC Study Team. Community-acquired pneumonia requiring hospitalization among U.S. children. N Engl J Med. 2015;372(9):835-845.

54. Kurz H, Göpfrich $H$, Huber $K$, et al. Spectrum of pathogens of in-patient children and youths with community acquired pneumonia: a 3 year survey of a community hospital in Vienna, Austria. Wien Klin Wochenschr. 2013;125(21-22):674-679.

55. Laundy M, Ajayi-Obe E, Hawrami K, Aitken C, Breuer J, Booy R. Influenza A community-acquired pneumonia in East London infants and young children. Pediatr Infect Dis J. 2003;22(10)(Suppl): S223-\$227.

56. Maltezou HC, La-Scola B, Astra H, et al. Mycoplasma pneumoniae and Legionella pneumophila in community-acquired lower respiratory tract infections among hospitalized children: diagnosis by real time PCR. Scand J Infect Dis. 2004;36(9):639-642.

57. Numazaki K, Chiba S, Umetsu M, et al. Etiological agents of lower respiratory tract infections in Japanese children. In Vivo. 2004;18(1): 67-71.

58. Tsolia MN, Psarras S, Bossios A, et al. Etiology of communityacquired pneumonia in hospitalized school-age children: evidence for high prevalence of viral infections. Clin Infect Dis. 2004;39(5): 681-686.

59. Graffelman AW, Willemssen FE, Zonderland HM, Neven AK, Kroes $A C$, van den Broek PJ. Limited value of chest radiography in predicting aetiology of lower respiratory tract infection in general practice. Br J Gen Pract. 2008;58(547):93-97.

60. Defilippi A, Silvestri M, Tacchella A, et al. Epidemiology and clinical features of Mycoplasma pneumoniae infection in children. Respir Med. 2008;102(12):1762-1768.

61. Hozo SP, Djulbegovic B, Hozo I. Estimating the mean and variance from the median, range, and the size of a sample. BMC Med Res Methodol. 2005;5:13.

62. Noah ND. Epidemiology of Mycoplasma pneumoniae infection in the United Kingdom: an analysis of reports to the Public Health Laboratory Service of England and Wales. Infection. 1976;4(1) (Suppl):25-28.

63. Lind K, Benzon MW, Jensen JS, Clyde WA Jr. A seroepidemiological study of Mycoplasma pneumoniae infections in Denmark over the 50-year period 1946-1995. Eur J Epidemiol. 1997;13(5):581-586. 
64. Park S, Lee SH, Seo KH, et al. Epidemiological aspects of pertussis among adults and adolescents in a Korean outpatient setting: a multicenter, PCR-based study. J Korean Med Sci. 2014;29(9):1232-1239.

65. Philipson K, Goodyear-Smith F, Grant CC, Chong A, Turner N, Stewart J. When is acute persistent cough in school-age children and adults whooping cough? A prospective case series study. $\mathrm{Br} J \mathrm{Gen}$ Pract. 2013;63(613):e573-e579.

66. Riffelmann M, Littmann M, Hülsse C, O'Brien J, Wirsing von König $\mathrm{CH}$. [Pertussis: incidence, symptoms and costs]. Dtsch Med Wochenschr. 2006;131(50):2829-2834.

67. Wang K, Fry NK, Campbell H, et al. Whooping cough in school age children presenting with persistent cough in UK primary care after introduction of the preschool pertussis booster vaccination: prospective cohort study. BMJ. 2014;348:g3668.

68. van den Brink G, Wishaupt JO, Douma JC, Hartwig NG, Versteegh FG. Bordetella pertussis: an underreported pathogen in pediatric respiratory infections, a prospective cohort study. BMC Infect Dis. 2014;14:526.

69. Harnden A, Grant C, Harrison T, et al. Whooping cough in school age children with persistent cough: prospective cohort study in primary care. BMJ. 2006;333(7560):174-177.

70. Diez-Domingo J, Ballester A, Baldó JM, et al. Incidence of pertussis in persons $<$ or $=15$ years of age in Valencia, Spain: seroprevalence of antibodies to pertussis toxin (PT) in children, adolescents and adults. J Infect. 2004;49(3):242-247.

71. Niederman MS, McCombs JS, Unger AN, Kumar A, Popovian R. The cost of treating community-acquired pneumonia. Clin Ther. 1998;20(4):820-837.

72. Centers for Disease Control and Prevention, National Center for Health Statistics. Pneumonia. http://www.cdc.gov/nchs/fastats/pneumonia.htm. Updated Jul 6, 2016.

73. Mandell LA, Wunderink RG, Anzueto A, et al; Infectious Diseases Society of America; American Thoracic Society. Infectious Diseases Society of America/American Thoracic Society consensus guidelines on the management of community-acquired pneumonia in adults. Clin Infect Dis. 2007:44(Suppl 2):S27-S72.

74. Dunbar LM, Khashab MM, Kahn JB, Zadeikis N, Xiang JX, Tennenberg AM. Efficacy of 750-mg, 5-day levofloxacin in the treatment of community-acquired pneumonia caused by atypical pathogens. Curr Med Res Opin. 2004;20(4):555-563.

75. Macfarlane J, Holmes W, Gard P, et al. Prospective study of the incidence, aetiology and outcome of adult lower respiratory tract illness in the community. Thorax. 2001;56(2):109-114.

76. Bisgard KM, Pascual FB, Ehresmann KR, et al. Infant pertussis: who was the source? Pediatr Infect Dis J. 2004;23(11):985-989.
77. Wendelboe AM, Njamkepo E, Bourillon A, et al; Infant Pertussis Study Group. Transmission of Bordetella pertussis to young infants. Pediatr Infect Dis J. 2007;26(4):293-299.

78. Klein NP, Bartlett J, Fireman B, Rowhani-Rahbar A, Baxter R, et al, Comparative effectiveness of acellular versus whole-cell pertussis vaccines in teenagers. Pediatrics. 2013;131(6):e1716-22.

79. Klein NP, Bartlett J, Rowhani-Rahbar A, Fireman B, Baxter R. Waning protection after fifth dose of acellular pertussis vaccine in children. N Engl J Med. 2012;367(11):1012-1019.

80. Koepke R, Eickhoff JC, Ayele RA, et al. Estimating the effectiveness of tetanus-diphtheria-acellular pertussis vaccine (Tdap) for preventing pertussis: evidence of rapidly waning immunity and difference in effectiveness by Tdap brand. J Infect Dis. 2014;210(6):942-953.

81. Wang K, Gill P, Perera R, Thomson A, Mant D, Harnden A. Clinical symptoms and signs for the diagnosis of Mycoplasma pneumoniae in children and adolescents with community-acquired pneumonia. Cochrane Database Syst Rev. 2012;10:CD009175.

82. Thom DH, Grayston JT, Campbell LA, Kuo CC, Diwan VK, Wang SP. Respiratory infection with Chlamydia pneumoniae in middleaged and older adult outpatients. Eur J Clin Microbiol Infect Dis. 1994;13(10):785-792.

83. Principi N, Esposito S. Emerging role of Mycoplasma pneumoniae and Chlamydia pneumoniae in paediatric respiratory-tract infections. Lancet Infect Dis. 2001;1(5):334-344.

84. Haubitz S, Hitz F, Graedel L, Batschwaroff M, Wiemken T4, Peyrani $P$, et al. Ruling out Legionella in community-acquired pneumonia. Am J Med. 2014;127(10):1010.e11-9.

85. Cornia PB, Hersh AL, Lipsky BA, Newman TB, Gonzales R. Does this coughing adolescent or adult patient have pertussis? JAMA. 2010;304(8):890-896.

86. van Vugt SF, Broekhuizen BD, Lammens C, et al; GRACE consortium. Use of serum $C$ reactive protein and procalcitonin concentrations in addition to symptoms and signs to predict pneumonia in patients presenting to primary care with acute cough: diagnostic study. BMJ. 2013;346:f2450.

87. Haran JP, Beaudoin FL, Suner S, Lu S. C-reactive protein as predictor of bacterial infection among patients with an influenza-like illness. Am J Emerg Med. 2013;31(1):137-144.

88. van Vugt SF, Broekhuizen BD, Zuithoff NP, et al; GRACE Consortium. Validity of a clinical model to predict influenza in patients presenting with symptoms of lower respiratory tract infection in primary care. Fam Pract. 2015;32(4):408-414. 